PROCEEDINGS OF THE

AMERICAN MATHEMATICAL SOCIETY

Volume 128, Number 5, Pages 1285-1292

S 0002-9939(99)05177-1

Article electronically published on August 3, 1999

\title{
IWASAWA INVARIANTS AND CLASS NUMBERS OF QUADRATIC FIELDS FOR THE PRIME 3
}

\author{
HISAO TAYA \\ (Communicated by David E. Rohrlich) \\ Dedicated to Professor Koji Uchida on his 60th birthday
}

\begin{abstract}
Let $d$ be a square-free integer with $d \equiv 1(\bmod 3)$ and $d>0$. Put $k^{+}=\mathbb{Q}(\sqrt{d})$ and $k^{-}=\mathbb{Q}(\sqrt{-3 d})$. For the cyclotomic $\mathbb{Z}_{3}$-extension $k_{\infty}^{+}$of $k^{+}$, we denote by $k_{n}^{+}$the $n$-th layer of $k_{\infty}^{+}$over $k^{+}$. We prove that the 3-Sylow subgroup of the ideal class group of $k_{n}^{+}$is trivial for all integers $n \geq 0$ if and only if the class number of $k^{-}$is not divisible by the prime 3 . This enables us to show that there exist infinitely many real quadratic fields in which 3 splits and whose Iwasawa $\lambda_{3}$-invariant vanishes.
\end{abstract}

\section{INTRODUCTION}

For a number field $k$ and a prime number $p$, we denote by $\lambda_{p}(k)$ the Iwasawa $\lambda$ invariant associated to the cyclotomic $\mathbb{Z}_{p}$-extension of $k, \mathbb{Z}_{p}$ being the ring of $p$-adic integers. When R. Greenberg visited Tokyo in May 1994, he asked the question whether there exist infinitely many real quadratic fields with $p$ split and $\lambda_{p}(k)$ vanishing, for a given odd prime number $p$. This is still an open problem.

Concerning the existence of infinite families of real abelian fields $k$ with $\lambda_{p}(k)=$ 0 , some results are well-known. For a number field $k$, we denote by $h(k)$ the class number of $k$. First, genus theory implies that for a given prime number $p$, there are infinitely many real cyclic fields $k$ of degree $p$ such that $p$ does not divide $h(k)$ and that $p$ does not split in $k$. Hence it follows from a theorem of Iwasawa Iw1 that there exist infinitely many real cyclic fields $k$ of degree $p$ with $\lambda_{p}(k)=0$. Further, Nakagawa and Horie gave in $[\mathrm{NH}$ a positive lower bound on the density of real quadratic fields $k$ such that the prime 3 does not divide $h(k)$ and that 3 does not split in $k$. Hence, in a similar way, there exist infinitely many real quadratic fields $k$ with $\lambda_{3}(k)=0$. Recently, Kraft $[\underline{\mathrm{Kr}}]$ also showed the existence of such an infinite family of real quadratic fields by using a result of Jochnowitz.

Results also exist for the case where the given prime may be split. In Iw3. page 185], for a given odd prime number $p$, Iwasawa gave a certain infinite family of real cyclic fields $k$ of degree $p$ with $\lambda_{p}(k)=0$ such that either $p$ divides $h(k)$ or $p$ splits in $k$. Also, for the prime 2, Ozaki and the author gave in OT] some

Received by the editors August 27, 1997 and, in revised form, June 22, 1998.

1991 Mathematics Subject Classification. Primary 11R23, 11R11, 11R29.

Key words and phrases. Iwasawa invariants, real quadratic fields, class numbers.

This research was partially supported by the Grant-in-Aid for Encouragement of Young Scientists, The Ministry of Education, Science, Sports and Culture, Japan. 
infinite families of real quadratic fields $k$ with $\lambda_{2}(k)=0$ such that either 2 divides $h(k)$ or 2 splits in $k$. Recently, Yamamoto $\mathrm{Ya}$ and Fukuda $\mathrm{Fu}$ gave results on the vanishing of $\lambda_{p}(k)$ for certain $(p, p)$-extensions and certain cyclic extensions of degree $p$, respectively, for an odd prime number $p$. These also enable us to give some infinite families of real abelian fields of type $(p, p)$, and of real cyclic fields of degree $p$, with $\lambda_{p}(k)=0$. However, for $p$ a fixed prime, we do not know whether there exist infinitely many real abelian fields $k$ with degree prime to $p$ such that $p$ splits completely in $k$ and $\lambda_{p}(k)=0$.

Let $\mathbb{Q}$ denote the field of rational numbers. In this paper, we first show the following theorem in Section 2. For the proof, we use a formula (Lemma 2 in Section 2) for the order of the $p$-Sylow subgroup of the ambiguous ideal class group in the cyclotomic $\mathbb{Z}_{p}$-extension of a real quadratic field in terms of a special value of a $p$-adic $L$-function (see also Remark 2 following the statement of Theorem 11).

Theorem 1. Let $d$ be a square-free integer with $d \equiv 1(\bmod 3)$ and $d>0$. Put $k^{+}=\mathbb{Q}(\sqrt{d})$ and $k^{-}=\mathbb{Q}(\sqrt{-3 d})$. For the cyclotomic $\mathbb{Z}_{3}$-extension $k_{\infty}^{+}$of $k^{+}$, denote by $k_{n}^{+}$the $n$-th layer in $k_{\infty}^{+} / k^{+}$and by $A_{n}^{+}$the 3 -Sylow subgroup of the ideal class group of $k_{n}^{+}$. Then $A_{n}^{+}$is trivial for all integers $n \geq 0$ if and only if the class number $h\left(k^{-}\right)$of $k^{-}$is not divisible by 3 .

Remark 1 . Let $d$ be a square-free integer with $d \not \equiv 1(\bmod 3)$ and $d>0$. Put $k^{+}=\mathbb{Q}(\sqrt{d})$ and $k^{-}=\mathbb{Q}(\sqrt{-3 d})$. In this case, Theorem 1 does not always hold. In fact, since 3 does not split in $k^{+}$, a theorem of Iwasawa [1w1] says that if $h\left(k^{+}\right)$ is not divisible by 3 , then $A_{n}^{+}$is trivial for all integers $n \geq 0$, even in the case where $h\left(k^{-}\right)$is a multiple of 3. For example, $h(\mathbb{Q}(\sqrt{29}))=1$ but $h(\mathbb{Q}(\sqrt{-3 \cdot 29}))=6$ for $d=29 \equiv 2(\bmod 3), h(\mathbb{Q}(\sqrt{69}))=1$ but $h(\mathbb{Q}(\sqrt{-3 \cdot 69}))=h(\mathbb{Q}(\sqrt{-23}))=3$ for $d=69 \equiv 6 \quad(\bmod 9)$, and $h(\mathbb{Q}(\sqrt{93}))=1$ but $h(\mathbb{Q}(\sqrt{-3 \cdot 93}))=h(\mathbb{Q}(\sqrt{-31}))=3$ for $d=93 \equiv 3(\bmod 9)$.

Remark 2. Theorem 1 can be also proved by a purely algebraic argument. This goes roughly as follows. Let $k^{+}, k^{-}$and $k_{\infty}^{+}$be as in Theorem 1, and let $k_{\infty}^{-}$be the cyclotomic $\mathbb{Z}_{3}$-extension of $k^{-}$. Denote by $M_{\infty}^{+}$the maximal abelian pro-3-extension of $k_{\infty}^{+}$unramified outside of the primes above 3 , and by $L_{\infty}^{-}$the maximal unramified abelian pro-3-extension of $k_{\infty}^{-}$. Then, by using the Kummer pairing [Iw2, $\S 7$ ] and [w2, Theorem 11], we see that $\operatorname{Gal}\left(M_{\infty}^{+} / k_{\infty}^{+}\right)$is pseudo-isomorphic to $\operatorname{Gal}\left(L_{\infty}^{-} / k_{\infty}^{-}\right)$, where $\dot{X}$ is $X$ with a twisted action of $\mathbb{Z}_{3}\left[\left[\operatorname{Gal}\left(k_{\infty}^{+} / k^{+}\right)\right]\right]$for a $\mathbb{Z}_{3}\left[\left[\operatorname{Gal}\left(k_{\infty}^{+} / k^{+}\right)\right]\right]$module $X$ (see [Iw2, page 278]). Since 3 splits in $k^{+}$, we find that $M_{0}^{+}$is an unramified extension over $k_{\infty}^{+}$, where $M_{0}^{+}$is the maximal abelian pro-3-extension of $k^{+}$unramified outside of the primes above 3 . Hence it follows from a certain relation between $M_{\infty}^{+}$and $M_{0}^{+}$(see [Iw2, $\left.\left.\S 3.2\right]\right)$ and Nakayama's lemma that $\operatorname{Gal}\left(L_{\infty}^{+} / k_{\infty}^{+}\right)$ is trivial if and only if $\operatorname{Gal}\left(M_{\infty}^{+} / k_{\infty}^{+}\right)$is trivial, where $L_{\infty}^{+}$is the maximal unramified abelian pro-3-extension of $k_{\infty}^{+}$. Since $\operatorname{Gal}\left(L_{\infty}^{-} / k_{\infty}^{-}\right)$and $\operatorname{Gal}\left(M_{\infty}^{+} / k_{\infty}^{+}\right)$have no nontrivial finite $\mathbb{Z}_{3}\left[\left[\operatorname{Gal}\left(k_{\infty}^{-} / k^{-}\right)\right]\right]$- and $\mathbb{Z}_{3}\left[\left[\operatorname{Gal}\left(k_{\infty}^{+} / k^{+}\right)\right]\right]$-submodules, respectively, we see by the above pseudo-isomorphism that $\operatorname{Gal}\left(L_{\infty}^{+} / k_{\infty}^{+}\right)$is trivial if and only if $\operatorname{Gal}\left(L_{\infty}^{-} / k_{\infty}^{-}\right)$is trivial. Therefore, the assertion of Theorem 1 follows immediately. Further, we mention here that Theorem 1 is also regarded as a special case of a recent result of Ozaki $\mathrm{Oz}$ Theorem 1]. Our proof given in Section 2 uses different methods.

Remark 3. Let $d$ be a square-free integer with $d>0$. Put $k^{+}=\mathbb{Q}(\sqrt{d})$ and $k^{-}=\mathbb{Q}(\sqrt{-3 d})$. Then, by the pseudo-isomorphism mentioned in Remark 2 we 
have $\lambda_{3}\left(k^{+}\right) \leq \lambda_{3}\left(k^{-}\right)$. In particular, if $\lambda_{3}\left(k^{-}\right)=0$, then $\lambda_{3}\left(k^{+}\right)=0$. However, in the case where $d \equiv 6(\bmod 9)$, we always have $\lambda_{3}\left(k^{-}\right) \geq 1$ because the 3 -adic $L$-function $L_{3}(s, \chi)$ has a trivial zero. Here $\chi$ is the non-trivial Dirichlet character associated to $k^{+}$. In such a case, Kraft [Kr] showed that if $\lambda_{3}\left(k^{-}\right)=1$, then $h\left(k^{+}\right)$ is not divisible by 3 , in particular $\lambda_{3}\left(k^{+}\right)=0$ (see also [S $\left(5_{B}^{\prime}\right)$ in Section 2]).

For a finite set $G$, we denote its cardinality by $\# G$. Theorem $\square$ and results of Nakagawa and Horie in [NH] enable us to give the following lower bound on the density of $k^{+}$such that $p$ splits in $k^{+}$and $A_{n}^{+}$is trivial for all integers $n \geq 0$. This is proved in Section 3.

Theorem 2. Let $k$ be a real quadratic field with discriminant $\Delta_{k}>0$. Denote by $k_{n}$ the $n$-th layer in the cyclotomic $\mathbb{Z}_{3}$-extension of $k$ and by $A_{n}$ the 3 -Sylow subgroup of the ideal class group of $k_{n}$. Then

$$
\liminf _{x \rightarrow \infty} \frac{\#\left\{k \mid \Delta_{k}<x, \Delta_{k} \equiv 1 \quad(\bmod 3), A_{n}=\{1\} \text { for all } n \geq 0\right\}}{\#\left\{k \mid \Delta_{k}<x\right\}} \geq \frac{3}{16} .
$$

By Theorem 2 we can give an affirmative answer for $p=3$ to the question mentioned in the beginning of this section. Namely, we obtain the following as a corollary.

Corollary 1. There exist infinitely many real quadratic fields $k$ with the prime 3 splitting in $k$ and with $\lambda_{3}(k)=\mu_{3}(k)=\nu_{3}(k)=0$, where $\mu_{3}(k)$ and $\nu_{3}(k)$ denote the Iwasawa $\mu$-and $\nu$-invariants, respectively, associated to the cyclotomic $\mathbb{Z}_{3}$-extension of $k$.

We also give in Section 3 an additional result on density in this direction (see Theorem 3).

Finally we mention some topics related to the problem treated here. It is natural to consider the problem whether there exist infinitely many prime numbers $p$ splitting completely in $k$ such that $\lambda_{p}(k)=0$ for a given real abelian field $k(\neq \mathbb{Q})$, by fixing the number field instead of fixing the prime number. This problem was also stated by Greenberg when he came to Tokyo. Recently, Ichimura [Ich] studied the problem and showed that for a certain real abelian field $k$, the problem can be solved if the $a b c$ conjecture for $k$ is valid. He also remarked that if $k$ is cyclic and its degree is a composite number, or if $k$ is non-cyclic, then the problem can be solved without assuming the $a b c$ conjecture.

There is a stronger conjecture on the Iwasawa invariants of totally real fields, known as Greenberg's conjecture, which states that both of $\lambda_{p}(k)$ and $\mu_{p}(k)$ always vanish for any totally real field $k$ and any prime number $p$ (cf. [Gr], also [Iw2, page 316]). Several authors gave a number of criteria for the validity of Greenberg's conjecture (for recent work, see the paper [IS] and its references), but the conjecture is at present unsolved except for the simplest case $k=\mathbb{Q}$. On the other hand, concerning $\mu_{p}$-invariants, it is well-known that $\mu_{p}(k)=0$ for any abelian field $k$ (not necessarily totally real) and any prime number $p$ by the theorem of Ferrero and Washington [FW].

\section{Proof of Theorem 1}

First, we recall some properties of $p$-adic $L$-functions. Let $p$ be an odd prime number and $\mathbb{C}_{p}$ the completion of the algebraic closure of the field of $p$-adic numbers. We denote by $\omega$ the Teichmüller character of $p$ and by $\chi$ a primitive even Dirichlet 
character of conductor $f_{\chi}$. Then the $p$-adic $L$-function $L_{p}(s, \chi)$ associated to $\chi$ is the unique $p$-adic meromorphic function from $\mathbb{Z}_{p}$ to $\mathbb{C}_{p}$ satisfying

$$
L_{p}(1-n, \chi)=-\left(1-\chi \omega^{-n}(p) p^{n-1}\right) \frac{B_{n, \chi \omega^{-n}}}{n}
$$

for all integers $n \geq 1$, where $B_{n, \chi \omega^{-n}}$ denotes, as usual, the generalized Bernoulli number (cf. Wa Theorem 5.11]). When $n=1$, they can be defined by

$$
B_{1, \chi \omega^{-1}}=\frac{1}{f_{\chi \omega^{-1}}} \sum_{a=1}^{f_{\chi \omega-1}} \chi \omega^{-1}(a) a
$$

where $f_{\chi \omega^{-1}}$ is the conductor of $\chi \omega^{-1}$. Assume that $\chi$ is non-trivial and that $f_{\chi}$ is not divisible by $p^{2}$. Then we have

$$
L_{p}(m, \chi) \equiv L_{p}(n, \chi) \quad(\bmod p)
$$

for any two integers $m$ and $n$, where both numbers can be shown to be $p$-integral (cf. Wa Corollary 5.13]). Further, if $f_{\chi}$ is prime to $p$, then $\chi \omega^{-n}(p)=0$. Hence we have the following.

Lemma 1. Let $p, \chi$ and $\omega$ be as above. Assume that $\chi$ is a non-trivial character with conductor prime to $p$. Then

$$
L_{p}(1, \chi) \equiv-B_{1, \chi \omega^{-1}} \quad(\bmod p) .
$$

On the other hand, letting $k$ be a real quadratic field, we know that there is a certain relationship between a $p$-adic $L$-function associated to $k$ and the $p$-parts of the ambiguous class groups in the cyclotomic $\mathbb{Z}_{p}$-extension of $k$. Namely, we showed the following fact in [Ta]:

Lemma 2 (Proposition 1 in [Ta]). Let $k$ be a real quadratic field with non-trivial Dirichlet character $\chi$ and let $p$ be an odd prime number. For the cyclotomic $\mathbb{Z}_{p^{-}}$ extension $k_{\infty}$ of $k$ with Galois group $\Gamma=\operatorname{Gal}\left(k_{\infty} / k\right)$, denote by $A_{n}$ the $p$-Sylow subgroup of the ideal class group of the $n$-th layer in $k_{\infty} / k$, and by $A_{n}^{\Gamma}$ the subgroup of $A_{n}$ consisting of ideal classes which are invariant under the action of $\Gamma$, namely, the p-part of the ambiguous class group of the $n$-th layer in $k_{\infty} / k$. Assume that $p$ splits in $k$. Then

$$
\# A_{n}^{\Gamma}=p^{v_{p}\left(L_{p}(1, \chi)\right)}
$$

for all sufficiently large integers $n$, where $v_{p}$ denotes the $p$-adic valuation normalized by $v_{p}(p)=1$.

Now we prove Theorem 1 We assume that $p=3$. Let $d$ be a square-free integer with $d \equiv 1(\bmod 3)$ and $d>0$, and put $k^{+}=\mathbb{Q}(\sqrt{d})$ and $k^{-}=\mathbb{Q}(\sqrt{-3 d})$, as in Theorem 1. Let $\chi$ be the non-trivial (even) Dirichlet character associated to $k^{+}$. Then $\chi \omega^{-1}$ is the non-trivial (odd) Dirichlet character associated to $k^{-}$. Since the conductor $f_{\chi \omega^{-1}}$ has at least two prime factors, we have $k^{-} \neq \mathbb{Q}(\sqrt{-1})$ and $k^{-} \neq \mathbb{Q}(\sqrt{-3})$. Hence the analytic class number formula (cf. [Wa, Theorem 4.17]) says that $h\left(k^{-}\right)=-B_{1, \chi \omega^{-1}}$. Therefore it follows from Lemma 1 that

$$
L_{3}(1, \chi) \equiv h\left(k^{-}\right) \quad(\bmod 3) .
$$

Let $\Gamma$ be the Galois group of $k_{\infty}^{+}$over $k^{+}$, and let $\left(A_{n}^{+}\right)^{\Gamma}$ be the subgroup of $A_{n}^{+}$ consisting of ideal classes which are invariant under the action of $\Gamma$. Since 3 splits 
in $k^{+}$, it follows from Lemma 2 that

$$
\#\left(A_{n}^{+}\right)^{\Gamma} \equiv 0 \quad(\bmod 3) \text { for sufficiently large } n \Longleftrightarrow L_{3}(1, \chi) \equiv 0 \quad(\bmod 3) .
$$

Therefore, combining (2.1) with (2.2), we see that $h\left(k^{-}\right)$is not divisible by 3 if and only if $\left(A_{n}^{+}\right)^{\Gamma}=\{1\}$ for all sufficiently large integers $n$. The latter assertion is equivalent to the assertion that $A_{n}^{+}=\{1\}$ for all integers $n \geq 0$, because $\left(A_{n}^{+}\right)^{\Gamma}=$ $\{1\}$ if and only if $A_{n}^{+}=\{1\}$ and because the primes lying above the prime 3 are totally ramified in $k_{\infty}^{+} / k^{+}$. This completes the proof of Theorem 1.

\section{Proof of Theorem 2}

First, we recall some results of Nakagawa and Horie in $\mathbf{N H}$. Let $m$ and $N$ be two positive integers satisfying the following condition:

(*) If an odd prime number $p$ is a common divisor of $m$ and $N$, then $p^{2}$ divides $N$ but not $m$. Further if $N$ is even, then (i) 4 divides $N$ and $m \equiv 1(\bmod 4)$, or (ii) 16 divides $N$ and $m \equiv 8$ or $12(\bmod 16)$.

For any integer $x \geq 0$, we denote by $K^{+}(x)$ the set of real quadratic fields with discriminant $\Delta_{k}<x$ and by $K^{-}(x)$ the set of imaginary quadratic fields with the absolute value of discriminant $\left|\Delta_{k}\right|<x$, and put

$$
\begin{aligned}
& K^{+}(x, m, N)=\left\{k \in K^{+}(x) \mid \Delta_{k} \equiv m \quad(\bmod N)\right\}, \\
& K^{-}(x, m, N)=\left\{k \in K^{-}(x) \mid \Delta_{k} \equiv m \quad(\bmod N)\right\} .
\end{aligned}
$$

Moreover, for a quadratic field $k$, we denote by $h_{3}^{*}(k)$ the number of ideal classes of $k$ whose cubes are principal. Then Nakagawa and Horie showed the following formulas for $h_{3}^{*}(k)$ in [NH, Theorem 1]:

$$
\begin{array}{lll}
\sum_{k \in K^{+}(x, m, N)} h_{3}^{*}(k) & \sim \frac{4}{3} \# K^{+}(x, m, N) & (x \rightarrow \infty), \\
\sum_{k \in K^{-}(x, m, N)} h_{3}^{*}(k) & \sim 2 \# K^{-}(x, m, N) & (x \rightarrow \infty),
\end{array}
$$

and the following estimate on $K^{ \pm}(x, m, N)$ in [NH] Proposition 2]:

$$
\# K^{+}(x, m, N) \sim \# K^{-}(x, m, N) \sim \frac{3 x}{\pi^{2} \varphi(N)} \prod_{p \mid N} \frac{q}{p+1} \quad(x \rightarrow \infty),
$$

where $X \sim Y(x \rightarrow \infty)$ means $\lim _{x \rightarrow \infty} \frac{X}{Y}=1$, and $\varphi$ is the Euler function, $\pi$ is the circular constant, and $p$ runs over all prime factors of $N$, and further, $q=4$ or $p$ according as $p=2$ or not. In particular, since $K^{ \pm}(x)=K^{ \pm}(x, 1,1)$, we have

$$
\# K^{+}(x) \sim \# K^{-}(x) \sim \frac{3 x}{\pi^{2}} \quad(x \rightarrow \infty)
$$

by (3.3).

Let us put

$$
\begin{aligned}
& K_{*}^{+}(x, m, N)=\left\{k \in K^{+}(x, m, N) \mid h_{3}^{*}(k)=1\right\}, \\
& K_{*}^{-}(x, m, N)=\left\{k \in K^{-}(x, m, N) \mid h_{3}^{*}(k)=1\right\} .
\end{aligned}
$$


Namely,

$$
\begin{aligned}
& K_{*}^{+}(x, m, N)=\left\{k \in K^{+}(x, m, N) \mid h(k) \not \equiv 0 \quad(\bmod 3)\right\}, \\
& K_{*}^{-}(x, m, N)=\left\{k \in K^{-}(x, m, N) \mid h(k) \not \equiv 0 \quad(\bmod 3)\right\} .
\end{aligned}
$$

Since

$$
\# K_{*}^{ \pm}(x, m, N)+3\left(\# K^{ \pm}(x, m, N)-\# K_{*}^{ \pm}(x, m, N)\right) \leq \sum_{k \in K^{ \pm}(x, m, N)} h_{3}^{*}(k),
$$

it follows that

$$
\# K_{*}^{ \pm}(x, m, N) \geq \frac{3}{2} \# K^{ \pm}(x, m, N)-\frac{1}{2} \sum_{k \in K^{ \pm}(x, m, N)} h_{3}^{*}(k) .
$$

Furthermore, concerning the right-hand side of the inequality above, we have

$$
\begin{array}{r}
\frac{3}{2} \# K^{+}(x, m, N)-\frac{1}{2} \sum_{k \in K^{+}(x, m, N)} h_{3}^{*}(k) \\
\sim \frac{5}{6} \# K^{+}(x, m, N) \quad(x \rightarrow \infty), \\
\frac{3}{2} \# K^{-}(x, m, N)-\frac{1}{2} \sum_{k \in K^{-}(x, m, N)} h_{3}^{*}(k) \\
\sim \frac{1}{2} \# K^{-}(x, m, N) \quad(x \rightarrow \infty),
\end{array}
$$

by (3.1) and (3.2).

Now, we prove Theorem 2 For a real quadratic field $k$, we denote by $k_{n}$ the $n$-th layer in the cyclotomic $\mathbb{Z}_{3}$-extension of $k$, and let $A_{n}$ be the 3-Sylow subgroup of the ideal class group of $k_{n}$ as in Theorem 2. First, Theorem 1 1tells us that we have a one-to-one correspondence between certain real and imaginary quadratic fields:

$$
\left\{k \in K^{+}(x, 1,3) \mid \# A_{n}=1 \text { for all } n \geq 0\right\} \longleftrightarrow K_{*}^{-}(3 x, 6,9)-\{\mathbb{Q}(\sqrt{-3})\}
$$

for any integer $x \geq 0$. Since $(m, N)=(6,9)$ satisfies condition $(*)$, it follows from (3.3), (3.4) and (3.6) that

$$
\liminf _{x \rightarrow \infty} \frac{\# K_{*}^{-}(3 x, 6,9)}{\# K^{-}(x)} \geq \lim _{x \rightarrow \infty} \frac{\# K^{-}(3 x, 6,9)}{2 \# K^{-}(x)}=\frac{3}{16} .
$$

Therefore, by (3.4) and (3.7), this implies that

$$
\liminf _{x \rightarrow \infty} \frac{\#\left\{k \in K^{+}(x, 1,3) \mid \# A_{n}=1 \text { for all } n \geq 0\right\}}{\# K^{+}(x)} \geq \frac{3}{16} .
$$

This completes the proof of Theorem [2,

Finally, we give the following theorem, which is a slight improvement of a result of Nakagawa and Horie $\mathrm{NH}$, Theorem 3].

Theorem 3. Let $k$ be a real quadratic field with discriminant $\Delta_{k}>0$. Then

$$
\liminf _{x \rightarrow \infty} \frac{\#\left\{k \mid \Delta_{k}<x, \lambda_{3}(k)=0\right\}}{\#\left\{k \mid \Delta_{k}<x\right\}} \geq \frac{17}{24} .
$$


Proof. A theorem of Iwasawa [Iw1] says that if $h(k)$ is not divisible by a prime $p$ not split in $k$, then the $p$-Sylow subgroup of the ideal class group in the cyclotomic $\mathbb{Z}_{p}$-extension of $k$ is trivial, in particular $\lambda_{p}(k)=0$. Using this, Nakagawa and Horie [NH, Theorem 3] showed the following:

$$
\liminf _{x \rightarrow \infty} \frac{\#\left\{k \mid \Delta_{k}<x, \Delta_{k} \not \equiv 1 \quad(\bmod 3), \lambda_{3}(k)=0\right\}}{\#\left\{k \mid \Delta_{k}<x\right\}} \geq \frac{25}{48} .
$$

In fact, by (3.3), (3.4) and (3.5), we have

$$
\begin{aligned}
& \liminf _{x \rightarrow \infty} \frac{\#\left\{k \mid \Delta_{k}<x, \Delta_{k} \equiv 2 \quad(\bmod 3), h(k) \not \equiv 0 \quad(\bmod 3)\right\}}{\#\left\{k \mid \Delta_{k}<x\right\}} \geq \frac{5}{16}, \\
& \liminf _{x \rightarrow \infty} \frac{\#\left\{k \mid \Delta_{k}<x, \Delta_{k} \equiv 3 \quad(\bmod 9), h(k) \not \equiv 0 \quad(\bmod 3)\right\}}{\#\left\{k \mid \Delta_{k}<x\right\}} \geq \frac{5}{48}, \\
& \liminf _{x \rightarrow \infty} \frac{\#\left\{k \mid \Delta_{k}<x, \Delta_{k} \equiv 6 \quad(\bmod 9), h(k) \not \equiv 0 \quad(\bmod 3)\right\}}{\#\left\{k \mid \Delta_{k}<x\right\}} \geq \frac{5}{48} .
\end{aligned}
$$

Therefore the desired result follows immediately from this and Theorem 2 This completes the proof of Theorem 3 ,

\section{ACKNOWLEDGMENT}

The author wishes to express his thanks to the referee for his corrections as well as for pointing out (see in Remark 2) the existence of a purely algebraic proof of Theorem 1.

\section{REFERENCES}

[FW] B. Ferrero and L. C. Washington, The Iwasawa invariant $\mu_{p}$ vanishes for abelian number fields, Ann. of Math. 109 (1979), 377-395. MR 81a:12005

[Fu] T. Fukuda, On the vanishing of Iwasawa invariants of certain cyclic extensions of $\mathbb{Q}$ with prime degree, Proc. Japan Acad. 73A (1997), 108-110. MR 98i:11091

[Gr] R. Greenberg, On the Iwasawa invariants of totally real number fields, Amer. J. Math. 98 (1976), 263-284. MR 53:5529

[Ich] H. Ichimura, A note on Greenberg's conjecture and the abc conjecture, Proc. Amer. Math. Soc. 126 (1998), 1315-1320. MR 98j:11093

[IS] H. Ichimura and H. Sumida, On the Iwasawa $\lambda$-invariants of certain real abelian fields II, International J. Math. 7 (1996), 721-744. MR 98e:11128c

[Iw1] K. Iwasawa, A note on class numbers of algebraic number fields, Abh. Math. Sem. Univ. Hamburg 20 (1956), 257-258. MR 18:644d

[Iw2] K. Iwasawa, On $\mathbb{Z}_{l}$-extensions of algebraic number fields, Ann. of Math. 98 (1973), 246-326. MR 50:2120

[Iw3] K. Iwasawa, A note on capitulation problem for number fields II, Proc. Japan Acad. 65A (1989), 183-186. MR 90k:11139

[Kr] J. S. Kraft, Class numbers and Iwasawa invariants of quadratic fields, Proc. Amer. Math. Soc. 124 (1996), 31-34. MR 96d:11112

[NH] J. Nakagawa and K. Horie, Elliptic curves with no rational points, Proc. Amer. Math. Soc. 104 (1988), 20-24. MR 89k:11113

[Oz] M. Ozaki, The class group of $\mathbb{Z}_{p}$-extensions over totally real number fields, Tohoku Math. J. 49 (1997), 431-435. MR 98g:11123

[OT] M. Ozaki and H. Taya, On the Iwasawa $\lambda_{2}$-invariants of certain families of real quadratic fields, Manuscripta Math. 94 (1997), 437-444. MR 99a:11122

[Ta] H. Taya, On cyclotomic $\mathbb{Z}_{p}$-extensions of real quadratic fields, Acta Arith. 74 (1996), 107119. MR 97c:11101 
[Wa] L. C. Washington, Introduction to Cyclotomic Fields, Graduate Texts in Math. Vol. 83. Springer-Verlag, New York, Heidelberg, Berlin 1982. MR 85g:11001

[Ya] G. Yamamoto, On the vanishing of Iwasawa invariants of certain $(p, p)$-extensions of $\mathbb{Q}$, Proc. Japan Acad. 73A (1997), 45-47. MR 98b:11111

Graduate School of Information Sciences, Tohoku University, 2-1-1 Katahira, SENDAI, 980-8577 JAPAN

E-mail address: taya@math.is.tohoku.ac.jp 